\title{
Respiratory infection of mice with mammalian reoviruses causes systemic infection with age and strain dependent pneumonia and encephalitis
}

\author{
Lianne Gauvin', Steffany Bennett ${ }^{1}$, Hong Liu', Mansoureh Hakimi ${ }^{3,4,5}$, Michael Schlossmacher ${ }^{3,4}$, Jay Majithia ${ }^{1}$
} and Earl G Brown ${ }^{1,2^{*}}$

\begin{abstract}
Background: Because mammalian reoviruses are isolated from the respiratory tract we modeled the natural history of respiratory infection of adult and suckling mice with T1 Lang (T1L) and T3 Dearing (T3D) reoviruses.

Methods: Adult and suckling Balb/c mice were infected by the intranasal route and were assessed for dose response of disease as well as viral replication in the lung and other organs. Viral antigen was assessed by immunofluorescence and HRP staining of tissue sections and histopathology was assessed on formalin fixed, $\mathrm{H}+\mathrm{E}$ stained tissue sections.

Results: Intranasal infection of adult mice resulted in fatal respiratory distress for high doses ( $10^{7} \mathrm{pfu}$ ) of T1L but not T3D. In contrast both T1L and T3D killed suckling mice at moderate viral dosages ( $10^{5} \mathrm{pfu}$ ) but differed in clinical symptoms where T1L induced respiratory failure and T3D caused encephalitis. Infections caused transient viremia that resulted in spread to peripheral tissues where disease correlated with virus replication, and pathology. Immunofluorescent staining of viral antigens in the lung showed reovirus infection was primarily associated with alveoli with lesser involvement of bronchiolar epithelium. Immunofluorescent and HRP staining of viral antigens in brain showed infection of neurons by T3D and glial cells by T1L.
\end{abstract}

Conclusions: These mouse models of reovirus respiratory infection demonstrated age and strain dependent disease that are expected to be relevant to understanding and modulating natural and therapeutic reovirus infections in humans.

Keywords: Mammalian reovirus, Pathogenesis, Pneumonia, Systemic infection, Intranasal infection, Serotype 3 Dearing, Serotype 1 Lang, Encephalitis, Immunofluorescence, Liver, Lung, Suckling mouse, Viremia

\section{Introduction}

Although mammalian orthoreovirus infection is not associated with disease, viruses are isolated from the respiratory and enteric tracts of humans and animals [1-4]. Natural infection with reoviruses may therefore involve either the nasal and/or oral routes of transmission. Recently serotype 3 strain Dearing (T3D) has been shown to possess oncolytic properties and is currently in clinical trials

\footnotetext{
* Correspondence: ebrown@uottawa.ca

'Department of Biochemistry, Microbiology and Immunology, Faculty of Medicine, University of Ottawa, 451 Smyth Rd, Ottawa, Ontario K1H 8M5, Canada

${ }^{2}$ Emerging Pathogens Research Centre, University of Ottawa, 451 Smyth Rd, Ottawa, Ontario K1H 8M5, Canada

Full list of author information is available at the end of the article
}

in humans raising fundamental questions regarding the natural history and normal patterns of reovirus infection in humans [5-7] as well as animal models.

Depending on the reovirus strain the suckling mouse can be infected by injection to cause encephalitis $[8,9]$ and myocarditis [10-12], and the oral route of infection has been shown to differ in effectiveness among reoviruses. In particular type1 strain Lang (T1L) and murine type 3 isolate Clone 9, can establish infection in suckling mice but not adult mice via the oral route [13-15], and high dosages of type 3 strain Dearing (T3D) are required for infection of mice via the oral route in both suckling and adult animals $[16,17]$.

\section{Biomed Central}


Although the prototype T1L and T3D viruses were initially isolated from stool samples of human infants, clinical specimens of reoviruses are routinely isolated from human respiratory tracts [1]. However, experimental data regarding mammalian reovirus respiratory infections are limited. Intranasal infection of adult volunteers has been demonstrated for all 3 serotypes of reoviruses (T1L, T2 Jones, and T3D) indicating that humans are infectable by the respiratory route using high dosages $\left(10^{7} \mathrm{pfu}\right)$ of virus with the induction of relatively mild clinical signs of respiratory infection for some patients [3]. The respiratory route, has been less well studied in the mouse, however models of T1L induced respiratory disease are described in specific strains of mice where CBA/J mice induced acute respiratory distress (ARD) which has been studied primarily from the standpoint of the immune responses to infection [18-20] but has also been shown to cause systemic spread via the blood to the spleen and intestine [21]. Pneumonia is also induced by T1L in other strains of mice (CD-1, Balb-c, and $\mathrm{C} 3 \mathrm{H})$ but with less severe pathology [22]. Although respiratory infection of mice with strains other than the prototype T1L reovirus has not been described, reovirus respiratory infection has been studied for T1L and T3D in adult rats which resulted in fatal pneumonia for T1L infections $[23,24]$. Recently lung infection of CBA/J mice via the intranasal route with T1L has been shown to be enhanced by prior uncoating with protease treatment [25].

Given that nasal infection of suckling mice has not been described for reoviruses we assessed the response of adult and suckling Balb-c mice to intranasal infection with T1L and T3D reoviruses. We found that both reoviruses establish infections in adult and suckling mice with high level replication in the lung as well as spread to involve peripheral organs. Fatal disease was associated with high dosage of T1L but not T3D in adult mice and with moderate dosages of T1L and T3D in suckling mice. The pattern of symptoms, viral replication and histopathology were consistent with fatal T1L pneumonia and T3D encephalitis. These data define the basic virological and pathological response to respiratory infection with reoviruses, indicating that both T1L and T3D can readily establish infection by the respiratory route but that these viral types differ in their tropism. T1L and T3D were pneumotropic but differed in extent. T3D also demonstrated fatal neurotropic infection following respiratory infection of new-born but not adult animals. Viral spread appeared to occur via the blood stream for both T1L and T3D viruses.

\section{Methods}

Viruses and cells

T1L and T3D prototype strains and L929 cells were originally obtained from Dr. B.N. Fields laboratory. Viruses were propagated and titrated in mouse L 929 cells grown in MEM plus 5\% fetal bovine serum and penicillin and streptomycin as described previously [26].

\section{Mouse infections and titrations}

Adult female 4-6 week old Balb-c mice (Charles River, St. Hyacinthe, Quebec) were anaesthetized with halothane (3\% in oxygen) before infection by application of virus suspended in $0.05 \mathrm{ml}$ PBS onto the nose pad. This method introduces virus into the lung as evident by distribution of trypan blue dye into lung tissues of anaesthetized mice that were euthanized without recovery from anaesthetization (data not shown). Suckling Balb-c mice were similarly infected at 2 days of age with $0.01 \mathrm{ml}$ volumes of virus. Survival was monitored for 30 days. Viral growth and pathology were determined on tissues collected from mice euthanized by $\mathrm{CO}_{2}$ narcosis. Blood was collected by cardiac puncture. Organs from duplicate animals were collected at each time point, suspended in 9 volumes of PBS and disrupted by sonication for 4 minutes on ice using a microprobe and the Model F60 Fisher Scientific sonicator at power setting $5-7$ before duplicate titrations by plaque assay in L929 cells to yield 2 technical replicate titrations for each experimental replicate. Infectious titres were calculated per gram of tissue. Data was plotted as mean values with variation shown as \pm 1 standard error.

\section{Animal ethics approval}

This study was carried out in accordance and compliance with the guidelines of the Canadian Council on Animal Care (CCAC) as outlined in the Care and Use of Experimental Animals, Vol.1, 2nd Edn. (1993), which are recognized as "best-practices" by the International Council for Laboratory Animal Science (ICLAS). The protocol was approved by the University of Ottawa Animal Care Committee (Protocol Number: BMI-85).

\section{Histopathology}

Adult mice were perfused with PBS and then formalin (3.75\% formaldehyde in PBS) before dissection and removal of organs for immersion in formalin for $24 \mathrm{hr}$, paraffin embedding, sectioning and hematoxylin and eosin staining. Suckling mice were not perfused before fixation but instead were dissected and placed in formalin for $24 \mathrm{hr}$ fixation with the exception of lungs that were inflated with formalin under $25 \mathrm{~cm}$ water pressure for 15 minutes before further fixation and processing as described for adult tissues. Horse radish peroxide (HRP) staining of formalin fixed paraffin embedded T1L and T3D infected brain tissues was done as described previously [27]. Images were collected using the 10X objective using an Olympus BX50 microscope. 


\section{Frozen sectioning and immunofluorescent staining}

Animals were sacrificed and perfused as described above before excising tissues that were snap frozen on dry ice in OCT compound (Fisher Scientific, Ottawa) for sectioning as previously described [28]. Tissue sections were fixed with cold acetone before immunofluorescent staining using the corresponding rabbit immune serum produced against purified T1L or T3D viruses. Immune sera were preadsorbed with acetone extracted, powdered mouse tissues $(10 \%(\mathrm{w} / \mathrm{v})$ tissue mixed with serum for $16 \mathrm{hr}$ at 4 C). Primary antibodies were diluted (1/800) in $0.3 \%$ BSA in PBS and incubated with sections for $0.5 \mathrm{hr}$ followed by $3 \times 5$ min washes in PBS. The secondary antibody was CY3 conjugated donkey anti rabbit IgG (1/800 dilution) (Jackson Labs, Maine) that was reacted as for the primary antibodies. After the last PBS wash the sections were incubated with DAPI $(1 \mathrm{ug} / \mathrm{ml})$ for 2 minutes to stain nuclei. Images were collected using an epifluorescent Leica
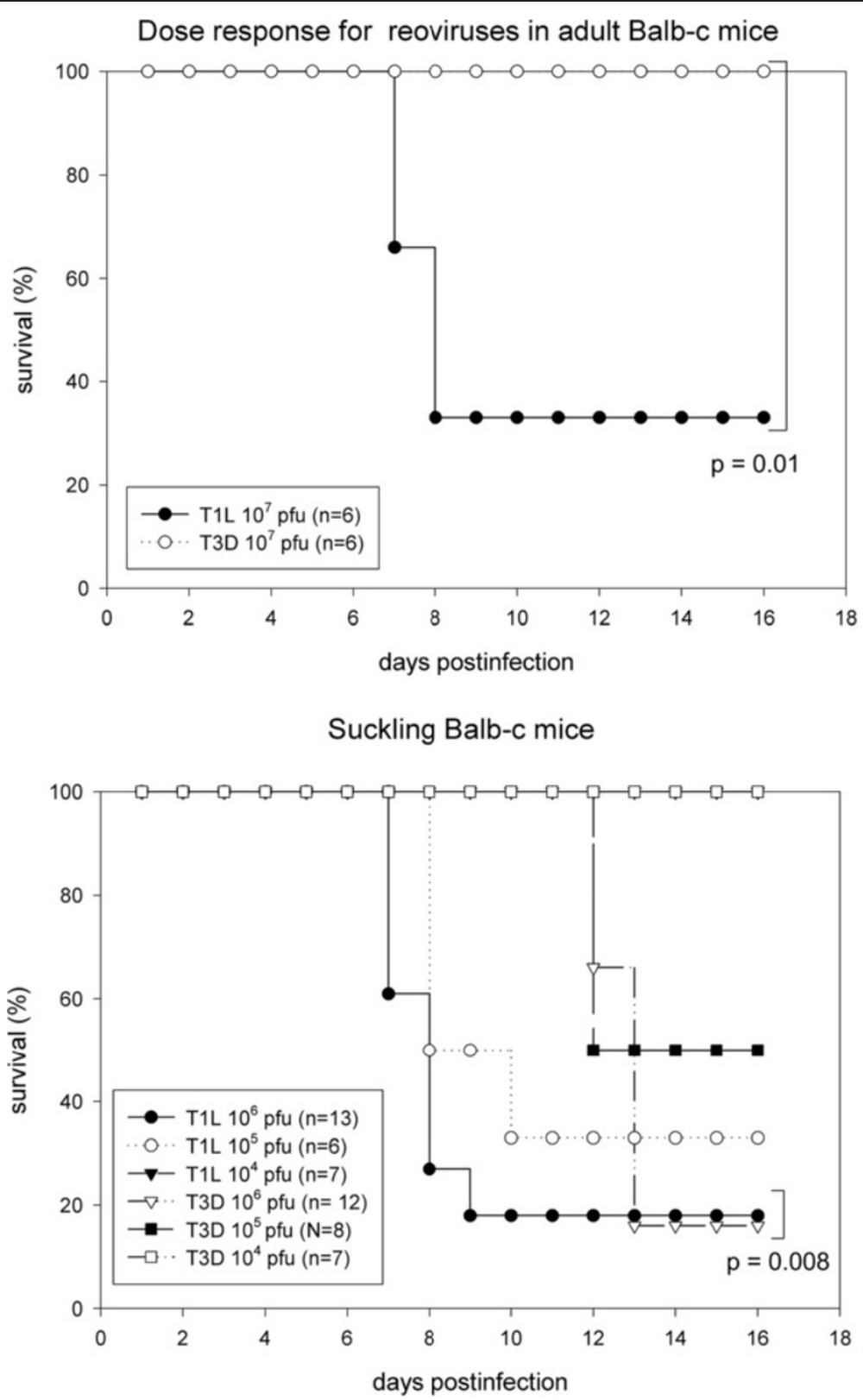

Figure 1 Age and virus dependent fatal dose response of reovirus T1L and T3D infection in adult and suckling Balb-c mice. The number of animals monitored in each group is shown as values of $\mathrm{n}$. Top panel. Adult mice were intranasaly infected with $10^{7} \mathrm{pfu}$ of either T1L or T3D and monitored for 30 days. Lower dosages did not induce fatality (not shown). Bottom panel. Suckling mice at 2 days of age were intranasaly infected with graded dosages of either T1 L or T3D and monitored for 30 days. The times to death were significantly different using the students $t$ test for the comparisons indicated in brackets. 
DMXRA 2 microscope (Leica Mississauga ON) using a Hamamatsu ORCA ER camera (Hamamatsu Corp., Bridgewater, NJ) running OpenLab v3.17 software (Improvision, Lexington, MA). Images were subsequently processed using Adobe Photoshop (Adobe Systems, San Jose CA).

\section{Statistical analysis}

Statistical analysis employed the single sample or paired $t$ test as indicated, using the Microsoft Office 2007 XL program.

\section{Results}

As the suckling mouse model of mammalian reovirus infection has been extensively studied following inoculation via the oral and injection routes, we set out to extend this analysis to the comparison of infection in adults and suckling mice following respiratory inoculation. Intranasal instillation of virus inoculum into anaesthetized mice results in the introduction of virus into the upper respiratory tract and lung.

\section{Age and virus dependent pneumonia and encephalitis in} response to intranasal infection

Adult Balb-c mice (4-6 wk old) were infected with graded dosages of $\mathrm{T} 1 \mathrm{~L}$ or $\mathrm{T} 3 \mathrm{D}$ virus and observed for 30 days. The highest dose of T1L $\left(1 \times 10^{7} \mathrm{pfu}\right)$ induced signs of disease and resulted in the death of 4 of 6 mice commencing 7 days post infection (Figure 1) that was associated with piloerection, hunching and respiratory distress that was consistent with pneumonia. The same dose of T3D did not induce any detectable signs of disease and resulted in a statistically significant increased survival (time of death greater than 16 days, $\mathrm{p}=0.01$ by student's t test) indicating a distinct difference in disease syndrome following respiratory infection with these viruses (Figure 1).

In contrast to adult mice, both $\mathrm{T} 1 \mathrm{~L}$ and $\mathrm{T} 3 \mathrm{D}$ viruses resulted in lethal infections in suckling mice with similar dose responses $\left(\mathrm{LD}_{50}\right.$ of $\left.10^{5} \mathrm{pfu}\right)$, however the time to death and the clinical symptoms of infection were significantly different ( $\mathrm{t}$ test, $\mathrm{p}=0.0082$ ) (Figure 1). Suckling mice infected with T1L developed respiratory crackling sounds on day 1 or 2 that progressed to severe respiratory distress and death by day 7 . Suckling mice infected with T3D also manifested respiratory crackling on day 2 but this resolved by day 8 and was followed by signs of neurological dysfunction and death commencing on day 11 . Neurological symptoms included tremors with darting and/or agitated behavior, spinning or turning, ataxia, and asymmetric gait. These experiments showed that respiratory infection with T1L and T3D not only induced different types of diseases in suckling mice but also differed in their ability to induce disease in adult mice.

\section{Viral replication in the lung and spread to peripheral organs}

Infection of adult mice with $10^{7} \mathrm{pfu}$ of T1L was associated with fatal disease in contrast to T3D that did not produce clinical disease, so it was not clear whether T3D was establishing an infection in adult mice. To further characterize reovirus respiratory infection and determine whether differences in ability to infect or replicate in tissues was responsible for the differences in disease response between these viruses, the level of infectious virus was determined for different tissues 2 days after infection with $10^{5}$ pfu of each virus. Both T1L and T3D yielded high infectious titres in the lung (approximately $10^{7} \mathrm{pfu} / \mathrm{g}$ ) and virus was observed to have spread to other tissues to yield 100-1000 fold lower levels of virus in the heart, liver, spleen, brain, kidney, blood and intestine (Figure 2). We cannot exclude the possibly that the virus detected in the intestinal samples was from infection via the gastric route as a consequence of respiratory inoculation versus dissemination from the lung and thus the significance of intestinal virus awaits further characterization. The presence of $10^{4} \mathrm{pfu} / \mathrm{g}$ virus in blood on day 2 following infection with either T1L or

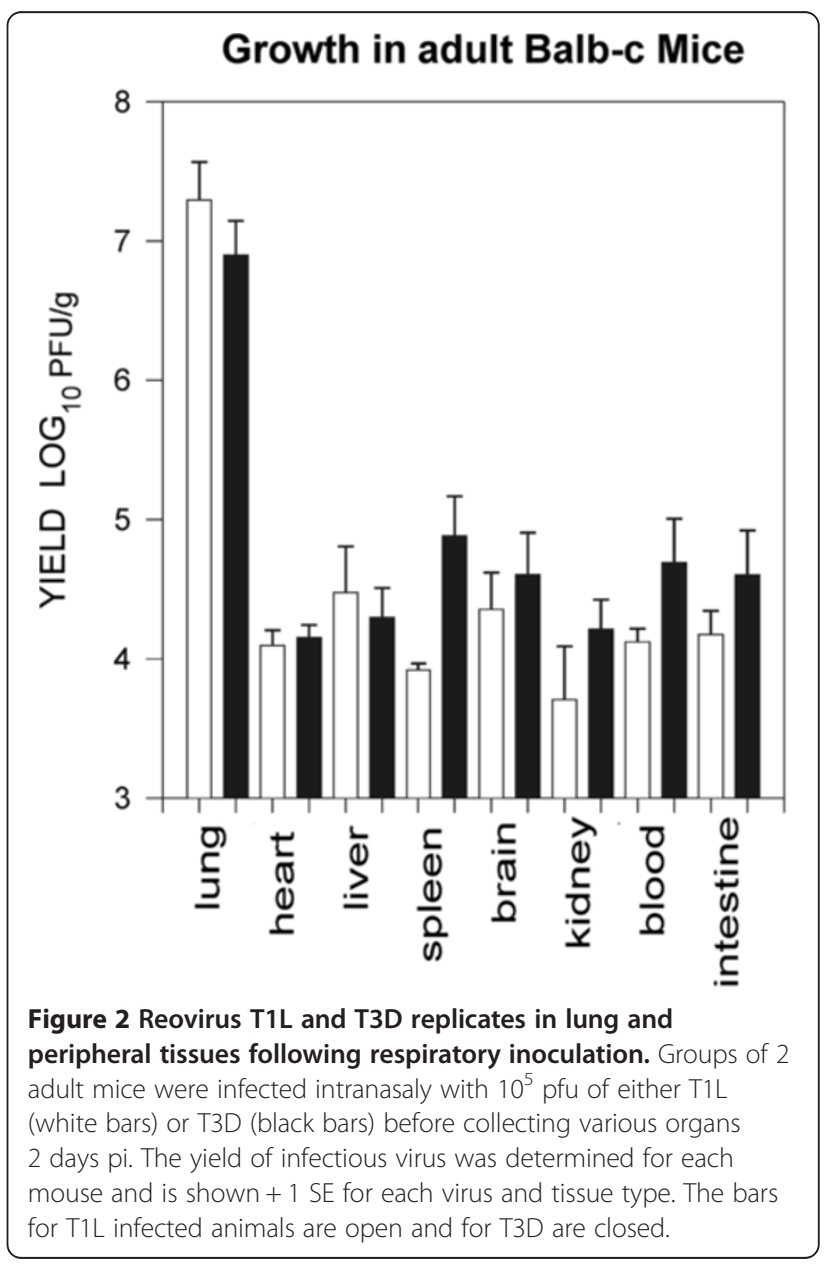


T3D indicated that reovirus was employing the hematogenous route for spread in the adult mouse.

To more fully assess the infection process, the time course of viral replication was measured in the lung as well as the brain and liver of adult mice. Growth of both T1L and T3D were similar for the first 2 days in lung tissues, at which time T3D had attained a maximum titre (Figure 3). T1L continued to replicate to reach a maximal titre on day 3 of $7.6 \times 10^{7} \mathrm{pfu}$, which was 10 fold higher than T3D. The higher yield of T1L than T3D infected mice was statistically significant when compared over days 3,5 , and 7 by paired $t$ test for $n=6$ samples $(p=0.0061)$. Replication in the liver and brain was to lower levels than the lung but was statistically similar for both viruses $(\mathrm{p} \geqq 0.05$ by paired $\mathrm{t}$ test $\mathrm{n}=$ 6-8). Virus was found in the blood for the first 2 and 3 days of infection for T3D and T1L respectively, after which infectious virus was undetectable $\left(<10^{2} \mathrm{pfu} / \mathrm{g}\right)$ (Figure 4).

The yield of infectious virus was also measured for suckling mice infected via the respiratory route with the same dose of virus as employed for adult mice $\left(10^{5} \mathrm{pfu}\right)$. In the lung and liver, reovirus $\mathrm{T} 1 \mathrm{~L}$ grew to significantly higher levels than T3D, $\mathrm{p}=0.0064$ and 0.0009 , for days 4-8 and 2-8 respectively (by paired t test, $n=6-8$ ) (Figure 3). In the brain T1L and T3D produced similar

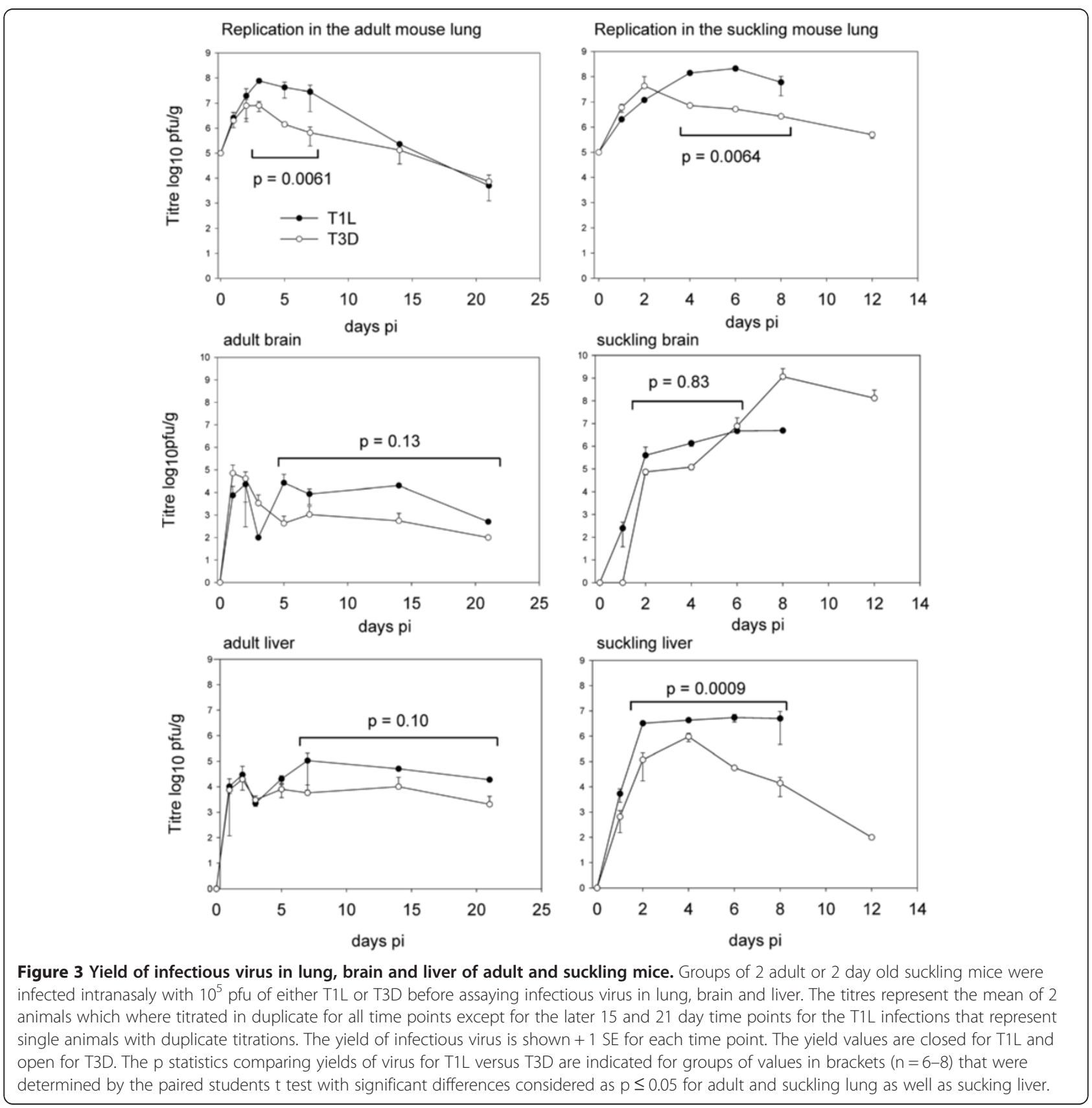




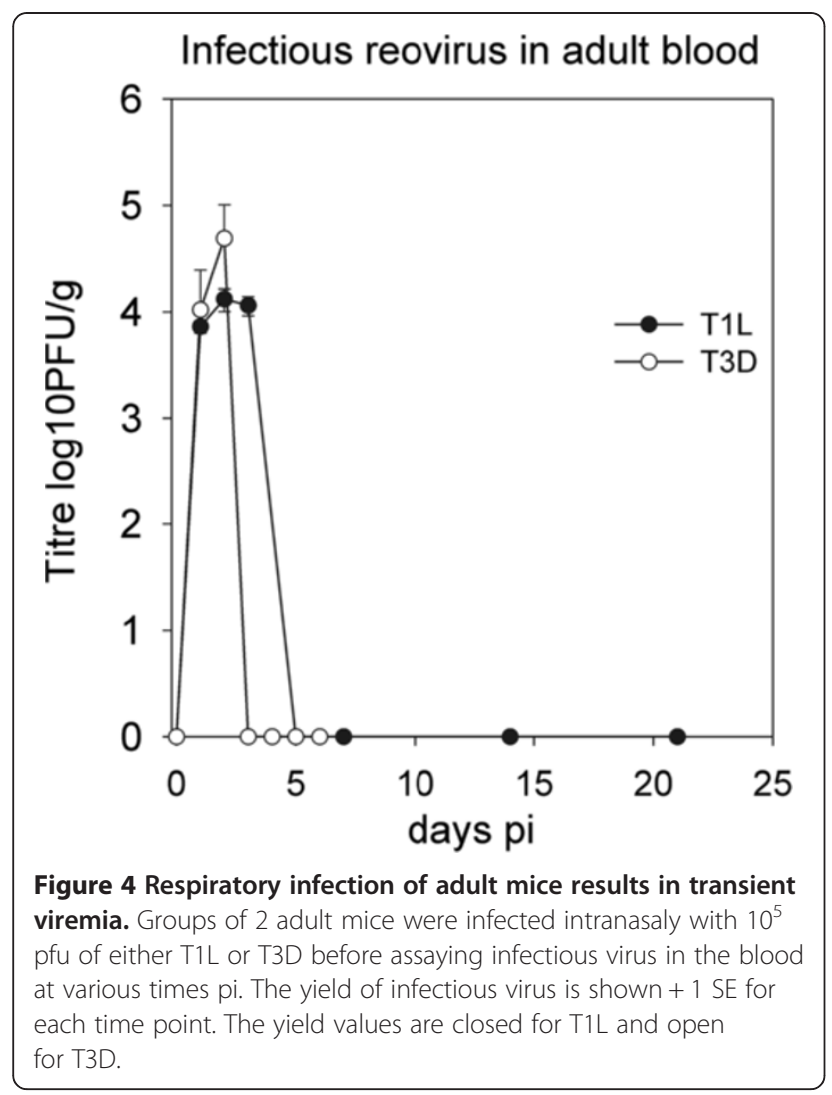

levels of virus until day 6 where after T3D grew to produce 1000 fold more virus on day 8 pi. Virus yield comparisons were not determined beyond day 8 because T1L produced lethal responses by day 7 pi. The high T3D titre in the brain was consistent with neurological signs of encephalitis in T3D infected suckling mice. The higher yield of T1L relative to T3D in suckling mouse lungs was also consistent with increased respiratory damage observed as respiratory distress. Both T1L and T3D grew to higher titres in the suckling mouse relative to the adult animal (Figure 3) which was consistent with their greater virulence in suckling animals.

\section{Immunofluorescent staining of mouse lungs demonstrated infection of alveoli}

Adult mice were infected with $10^{7}$ pfu of both strains of reovirus and monitored for viral antigen production in the lungs by immunofluorescent staining using typespecific antivirus rabbit serum (Figure 5). One day after infection viral antigen was prominent in lungs as multiple small patches of infection in the alveolar regions for both T1L and T3D infections. The number and size of infected sites had increased by day 2 (shown enlarged in Figure 6) but stabilized or decreased by day 3. The extent of viral staining reflected the viral growth in lungs where peak viral load and antigen staining were seen at days 2 and 3 for T3D and T1L respectively. There were very few patches of viral antigen present in bronchial epithelium which was primarily seen as individual cells or small patches of infected cells suggesting that bronchial epithelium was not a major site of infection for either T1L or T3D reoviruses.

The pattern of infection in suckling mouse lungs was very similar to adults except that more lung tissue was infected at day 3 for both virus types (Figure 7). Again the infection was largely alveolar with few infected epithelial cells, where small patches of epithelial infection were seen for T3D at day 3. Consistent with the reductions in staining after the times of peak virus yield, fluorescent antibody staining of lungs at 7 days pi showed low levels of staining of viral antigen, which was mainly associated with accumulated debris in the alveolar and bronchiolar airways in both adult and suckling mice infected with both reovirus T1L and T3D (data not shown).

\section{Immunofluorescent staining of brain}

We also performed fluorescent antibody staining for brains of suckling and adult animals on day 7 pi. The brains of adult mice did not contain detectable levels of viral antigen which was consistent with the low levels of infectious virus at this time (data not shown). Infection of the suckling brain with T3D resulted in clusters of fluorescent cells, as shown in Figure 8a. These cell were shown to be infected neurons (including Purkinje cells of the cerebellum) with the presence of antigen in cell bodies as well as processes as shown in Figure $8 \mathrm{~b}$ (single neuron in inset). Although brain infection with T1L was much reduced there were small areas of staining in the brainstem regions that involved non-neuronal cells with morphologies similar to activated glia (shown in Figure 8b). Staining of endothelial cells was also seen in small arterioles and capillaries of both T1L and T3D infected brains (as shown in the inset panel of the T1L infected brain, Figure 8b). Although select neuronal infection was also recorded in the hippocampus and cortex of T3D inoculated mice, further characterization is required to assess the cellular tropism and full extent of brain infection with both these viruses via the respiratory route.

Similar to the brain data, the livers of adult mice were negative by immunofluoresent staining at day 7 pi for T1L and T3D antigen (data not shown). Staining of uninfected mouse organs with anti-reovirus serum revealed no signal, as expected (data not shown). In contrast, staining of reovirus antigen at the same time point was seen in liver sections of suckling mice although this involved only a small number of hepatocytes that were more easily detectable in T1L infected than T3D infected livers (data not shown). Therefore the ability to detect antigen-positive cells in suckling brain and liver relative to adults was correlated with greater replication of mammalian reoviruses in suckling tissues than adult organs. 


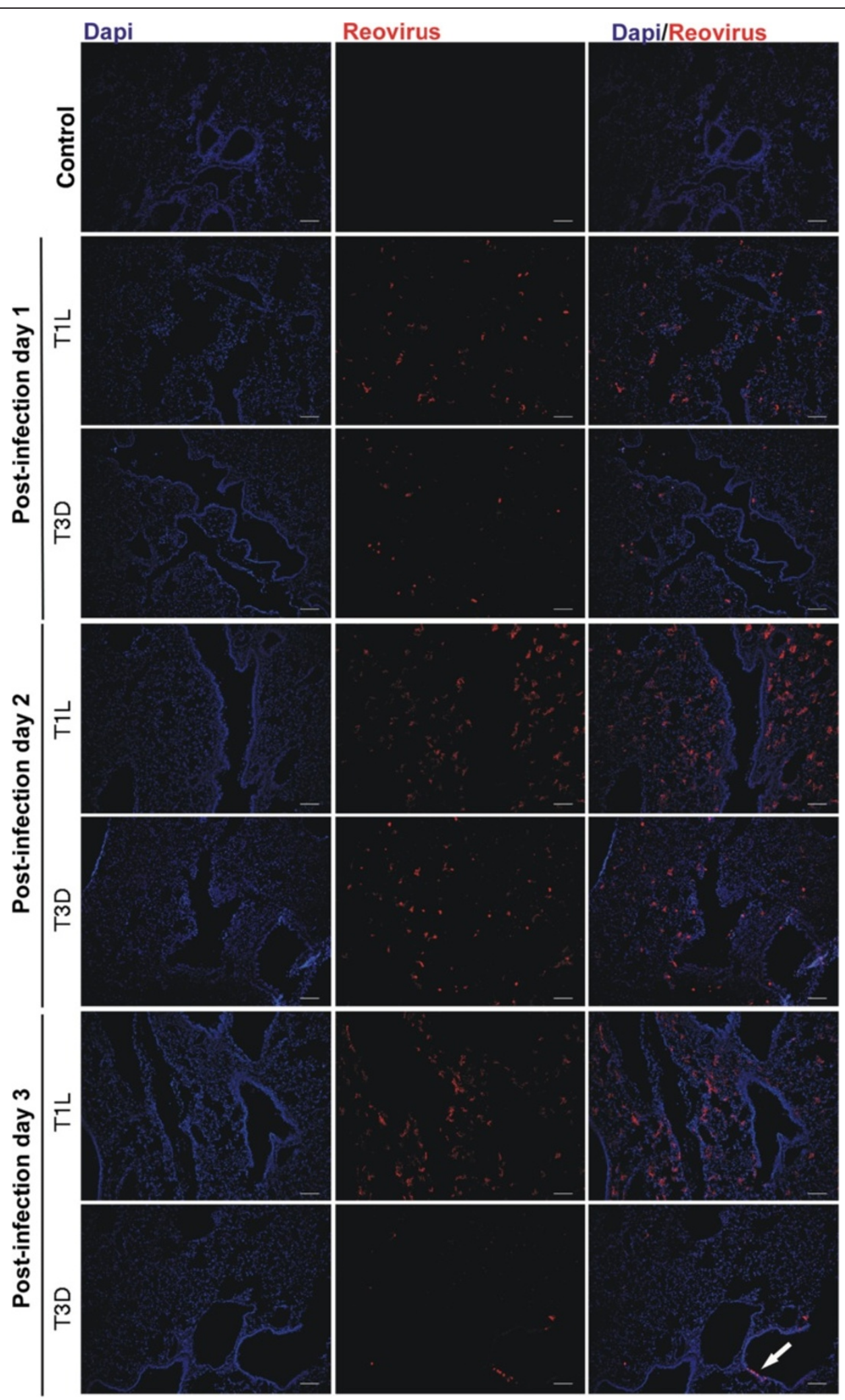

Figure 5 (See legend on next page.) 


\section{Histopathology}

We had demonstrated that respiratory inoculation with reovirus not only resulted in local infection in lung tissue but also spread to peripheral tissue sites. To assess the gross pathological consequences of infection, organs were paraffin embedded, sectioned and stained with hematoxylin and eosin at the acute stage of fatal infections; day 7 for all tissues except suckling brain that was analyzed at 13 days pi. Adult mice were infected intranasaly with $10^{7} \mathrm{pfu}$ and suckling mice with $10^{5}$ pfu of both T1L and T3D for histopathological assessment of adult brain, lung, heart, liver, spleen, kidney, intestine and suckling, lung, brain and liver. The histological observations were consistent with the virological findings in that organs with higher viral replication $\left(\geq 10^{6} \mathrm{pfu} / \mathrm{g}\right.$ ) revealed greater signs of pathology, which were detected as tissue damage and inflammation (i.e. accumulations of lymphocytes and inflammatory cells) for both T1L and $\mathrm{T} 3 \mathrm{D}$ in lungs and livers and for $\mathrm{T} 3 \mathrm{D}$ in sections of infected suckling brain, as shown in Figure 9. The adult and suckling lungs infected with T1L produced signs of severe patchy pneumonia associated with alveolar thickening and lymphocytic infiltration, as well as accumulation of cellular debris, with distended bronchioles and alveoli (Figure 9 panels b, c, e, and f relative to controls panels a and d). Lung infection with T3D was similar to T1L except that the degree of pathology, including areas of lymphocytic infiltration was reduced, consistent with a non-fatal primary viral pneumonia. Alveolar inflammation and thickening was more pronounced not only in suckling mice relative to adult animals but also for $\mathrm{T} 1 \mathrm{~L}$ relative to $\mathrm{T} 3 \mathrm{D}$ infection.

Infection of the liver of adult mice was associated with some minor foci of necrosis and inflammatory cells primarily associated with the vasculature and biliary ducts (data not shown). This pattern was more extensive in suckling mice, where T1L infection was associated with detectable areas of necrotic lesions and inflammatory cells, as indicated with arrows in panels $h$ and $j$ of Figure 9, relative to control sections shown in Figure 9g.

The brains of adult mice infected with T1L and T3D were not significantly different from control mice at 7 days pi (data not shown). Brains of suckling mice were sectioned 13 days after infection, corresponding to the time of neurological disease symptoms in T3D infection. Brains of T1L infected suckling mice appeared normal however the brains of T3D infected suckling mice revealed areas of encephalitis associated with the hippocampus and cortical regions seen as neurons with condensed nuclei, as indicated by an arrow in panel 1 of Figure 9. The histopathological findings for these organs as well as the heart, intestine and spleen are summarized in Table 1 . The heart and intestine appeared relatively normal, but the spleen was activated and possessed multiple follicles and white pulp. In summary, significant and severe pathology was restricted to the lungs of all mice infected with T1L and T3D viruses and the brain of T3D infected suckling mice.

\section{Discussion}

In this paper we extend the mouse models of reovirus infection to the respiratory tract where we demonstrate that both adult and suckling mice are readily infected via intranasal inoculation with prototype T1L and T3D reoviruses. Intranasal inoculation resulted in extensive lung infection that became systemic within the first day of infection. The pattern of disease was both type and age specific where T1L caused fatal, acute respiratory distress and T3D did not. In contrast, T3D produced an age-dependant fatal encephalitis in newborn but not adult mice. This indicated a uniform ability of both serotypes to initiate infection via the respiratory route but the resulting infections induced strain dependent polymorphisms with respect to extent of tissue involvement and disease type.

\section{The adult mouse model of respiratory reovirus infection} The overall adult mouse lung model of respiratory infection was characterized by local replication in alveolar epithelium coincident with appearance of virus in the blood stream. Within the first day of infection, blood borne virus infected peripheral organs to produce lower

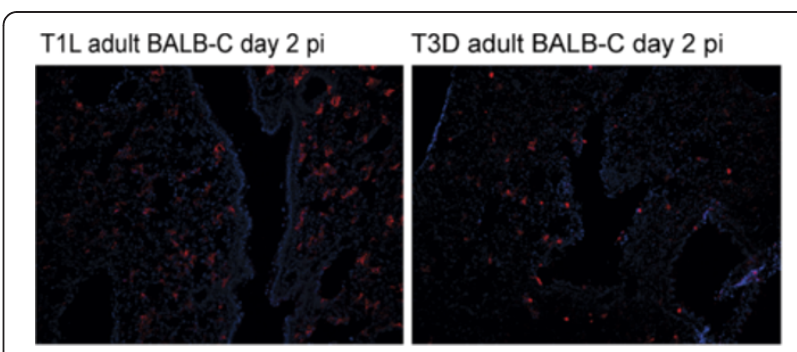

Figure 6 High magnification images of T1L and T3D infected adult lungs. The 2 day post infection images shown in Figure 5 are enlarged here to demonstrate more clearly the presence of reovirus antigens in alveolar tissues. Size bars are $100 \mu \mathrm{M}$. 


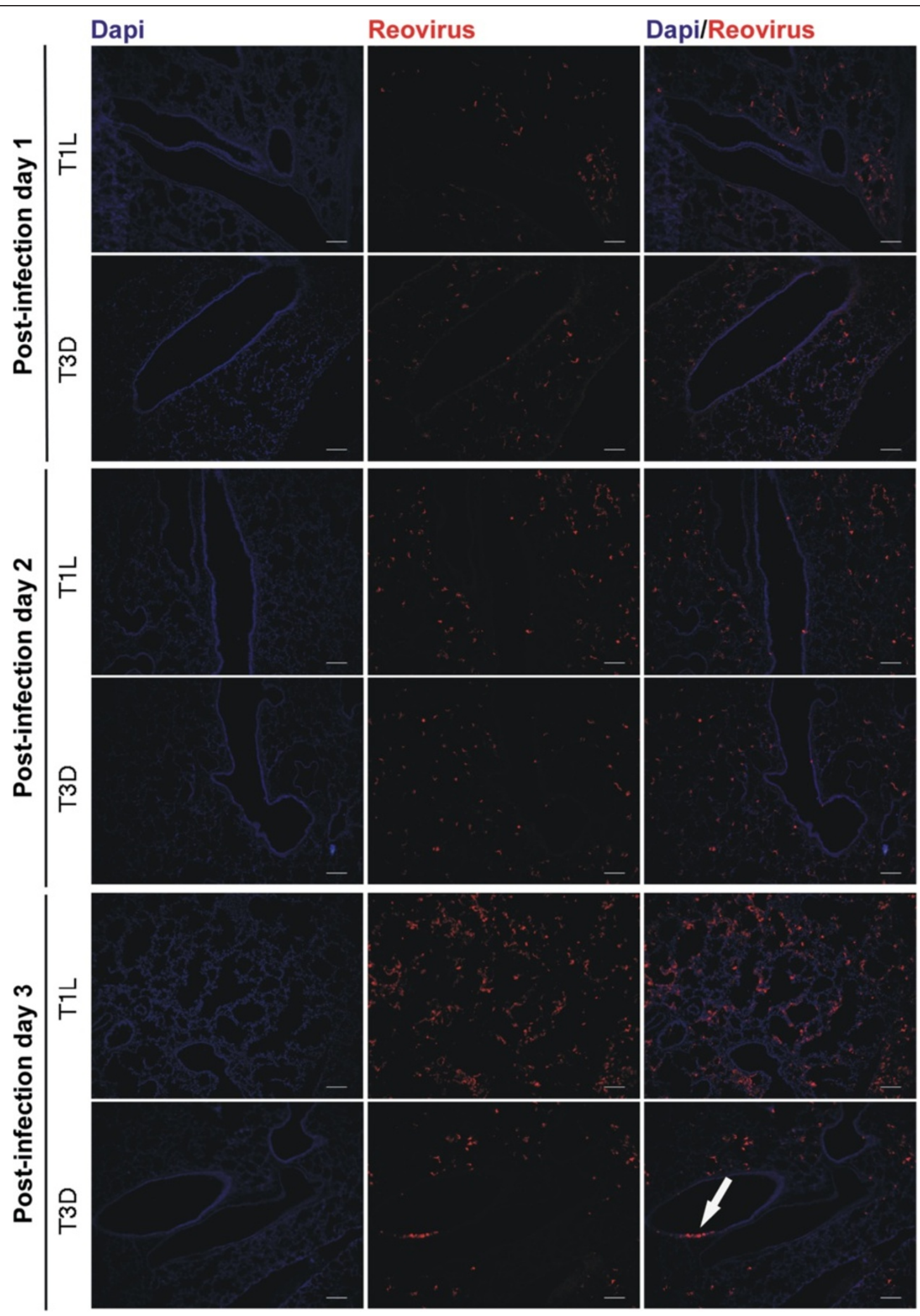

Figure 7 (See legend on next page.) 
(See figure on previous page.)

Figure 7 Time course of immunofluorescent staining of virus infected suckling lung. Two day old suckling mice were infected intranasaly with $10^{5}$ pfu of either T1L or T3D before fluorescent antibody staining of frozen lung sections after 1, 2, or 3 days of infection. T1L and T3D antigens were stained with virus specific antiserum as described in Figure 5. Cell nuclei were stained with DAPI, shown in blue. Size bars are $100 \mu \mathrm{M}$. Sagital sections show viral infection patterns similar to those of adults ( Figures 5 and 6 ) that again show sparse staining of epithelium in the bronchiolar airways (arrow) being restricted primarily to T3D infection.

levels of infectious virus that were not associated with disease states. T1L was more pneumotropic than T3D and caused dose dependent fatal acute respiratory distress which occurred 4 days subsequent to the time of peak virus yield in the lung and thus appeared to be largely due to an immune mediated inflammatory response (as shown for T1L in the CBA/J mouse [20]). T3D does not replicate as well as T1L in the mouse lung (Figure 3) consistent with the findings of others in the rat lung [23].

\section{The suckling mouse model of respiratory reovirus infection}

The suckling mouse lung model of respiratory infection was similar to the adult model for T1L but differed for

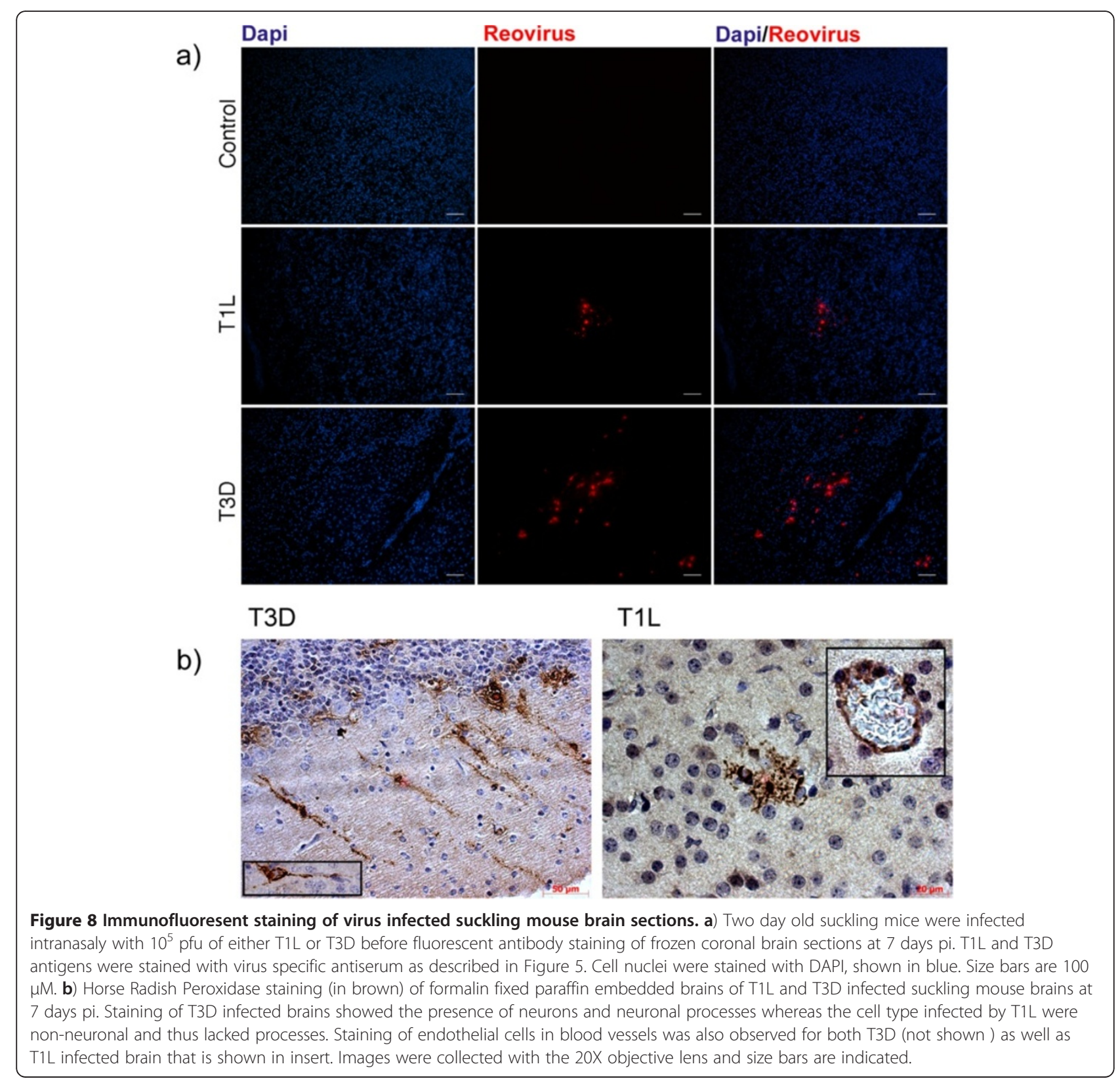





Figure 9 Pathology of virus infected lung, liver and brain tissues. Adult or 2 day old suckling mice were infected intranasaly with $10^{7}$ or $10^{5}$ pfu respectively with either T1L or T3D before sectioning formalin fixed, paraffin embedded sections for histopathological assessment by $\mathrm{H}$ and $\mathrm{E}$. staining. Panels $\mathbf{a}, \mathbf{d}, \mathbf{g}$, and $\mathbf{j}$ show uninfected control images for adult or suckling lung, liver and brain sections of mice as labeled. Panels $\mathbf{b}, \mathbf{e}, \mathbf{h}$, and $\mathbf{k}$ show the T1L infected lung, liver and brain of the indicated types (suckling or adult) of infected mouse organs. Panels $\mathbf{c}, \mathbf{f}, \mathbf{i}$, and $\mathbf{I}$ show the T3D infected lung, liver and brain for the indicated types (suckling or adult) of mouse organs. Arrows show local areas of cellular damage in liver and suckling brain sections that show the dentate gyrus of the hippocampus. Lungs and liver were imaged 7 days pi and brains at 13 days pi. Images were captured with the 20X objective.

T3D. Although both types replicate in the lung, T1L was more pneumovirulent than T3D causing pneumonia in both suckling and adult mice. T3D produces clinical signs of respiratory infection that were not fatal but instead resulted in dose dependent fatal encephalitis that occurred on day 12 , subsequent to maximal virus replication ( $>10^{9} \mathrm{pfu} / \mathrm{gram}$ of brain) around day 8 . The occurrence of disease subsequent to maximal virus yield again suggests a post-infectious host response to the virus as the cause of disease. Minor tissue necrosis was seen in the T1L infected liver and less so in T3D infection, which is consistent with the observation that the liver is the only site of tissue damage in the adult SCID model of intraperitoneal reovirus infection which is associated with systemic infection and hepatitis [29].

\section{Reovirus grows to high titre in lungs}

With respect to organ tropism, both $\mathrm{T} 1 \mathrm{~L}$ and $\mathrm{T} 3 \mathrm{D}$ strains infected the lung and all peripheral tissues examined, but T1L replicated to higher levels in the lung that was associated with its greater ability to cause acute respiratory distress in the mouse; these findings parallel those found by others in rats [23]. The clinical involvement of T1L infection with the lung was supported by the high level of infectious virus and infected lung tissue as well as the presence of interstitial alveolar inflammation. Both reovirus types grew to higher levels per gram of tissue in the suckling mouse lung that was associated with increased pneumovirulence of T1L but not T3D. Similar to adults, fatally infected T1L infected suckling mice demonstrated a time to death of 7 to 8 days, 
Table 1 Histopathological assessments of adult and suckling mice following intranasal infection

\begin{tabular}{|c|c|c|}
\hline Tissue & $\mathrm{T} 1 \mathrm{~L}$ virus infection & T3D virus infection \\
\hline Adult Lung & $\begin{array}{l}\text { severe patchy pneumonia, alveolar debris, lymphocytic infiltration, } \\
\text { distended bronchioles and alveoli }\end{array}$ & $\begin{array}{l}\text { mild to moderate pneumonia, discrete } \\
\text { lymphocytic infiltration }\end{array}$ \\
\hline Adult brain & appears normal & appears normal \\
\hline Adult liver & $\begin{array}{l}\text { few necrotic foci with cell degeneration and } \\
\text { polymorphonuclearleukocytes }\end{array}$ & edema, necrotic foci (fewer than T1L) \\
\hline Adult heart & appears normal & appears normal \\
\hline Adult spleen & numerous white follicles & numerous white follicles \\
\hline Adult intestine & appears normal & appears normal with some lymphocytes \\
\hline Suckling lung & severe pneumonia as seen for the adult infection & moderate pneumonia \\
\hline Suckling brain & minor areas of lymphocytes & encephalitis, dark staining condensed cells \\
\hline Suckling liver & necrotic foci and lymphocytes & less numerous necrotic foci and lymphocytes \\
\hline
\end{tabular}

however these infections differed in dose response where fatal disease occurred with at a 100 fold reduced dose $\left(10^{5}\right.$ versus $\left.10^{7} \mathrm{pfu}\right)$. In contrast, suckling mice infected with similar doses of T3D lived beyond this time to subsequently die of neurologic disease.

\section{Lung infection primarily involved alveolar tissues}

Cellular tropism in the lung was largely restricted to alveolar tissue seen as dispersed foci of infected cells similar to the infection of type I alveolar pneumocytes described for infected rat lungs [23]. Very little evidence of infection was seen for bronchiolar epithelium which was primarily detectable at later times, coincident with peak replication. The observation of alveolar rather than bronchiolar infection is opposite to the pattern seen for mouse infection with unadapted human viruses that produce local infections of the lung, i.e. influenza $[28,30,31]$ and parainfluenza viruses [32,33]. Previous analysis of infection of mouse enteric epithelium indicate entrance of virus through the apical side of $\mathrm{M}$ cells [34] followed by infection of the basolateral epithelial surfaces that contain 2 known reovirus receptors, sialic acid and junction adhesion molecule [35]. Entry into the rat lung has also been shown to involve transport through M cells [24]. Proteases present in mouse lung tissue may also promote infection by generating partially uncoated virions that can enter though $\mathrm{M}$ cells [25] where virus could subsequently disseminate to alveolar and peripheral organ sites for productive infection, possibly via infected endothelial cells in blood capillaries as was seen in infected brain (Figure 8b). Determination of the mechanistic details of infection and spread via the respiratory route in mice requires further analysis.

\section{Respiratory reovirus infection resulted in systemic spread via the blood}

Spread of virus in the suckling mouse has previously been shown to involve blood $[4,36]$ as well as neurons, with T3D employing primarily the neuronal route and T1L the hematogenous route [37]. Our data are consistent with entrance of virus into the blood from the lung followed by systemic spread. The spread of virus to the liver in the first day of infection (Figure 3) must be via a fast route such as blood circulation since T3D virus can only travel $14 \mathrm{~mm}$ per day through nerves $[37,38]$. Transmission of virus via nerves may however also be operating in the respiratory suckling mouse model as T3D virus was not detected in brain until day 2 suggesting a slower mode of spread to this site of infection, possibly from the nasal cavity via the olfactory nervous system. There may also be differences in infection and/or transport of T1L versus T3D through blood vessels because T1L has been shown to have an increased ability to replicate in endothelial cells [39]. Previous analysis of intracranial injection of suckling mice showed that T1L infection was limited to ependymal cells surrounding the choroids plexus that induced fatal obstructive hydrocephalus [36,40], however we observed T1L antigen positive cells in the brain parenchyma within structures suggestive of glia (Figure 8b). This was in marked contrast to T3D that caused neuronal infection and encephalitis that was consistent with oral and intracranial infection previously reported for suckling mice $[8,41]$. Future studies are needed to determine the sequence of events involved in the entry and spread of reovirus from the mouse respiratory tract to peripheral tissues.

\section{Reovirus oncolysis}

The ability of T3D to preferentially replicate in RAS transformed 3T3 cells has identified reovirus as an oncolytic virus $[6,7]$ that has now been demonstrated to replicate in a variety of tumor types as well as treat tumors in vivo models [5,42-44]. Treatment of tumors with oncolytic viruses is a process of applied pathogenesis where viral infection is directed at tumor destruction rather than disease production. It is therefore necessary to 
know the pathogenesis of reovirus infection for all tissue types as a consequence of infection by various routes. A thorough understanding of reovirus infection via the respiratory route is thus of practical importance in defining the ability of reovirus to infect human tissues and cause disease or conversely for the application of reovirus to the treatment of cancer. The mouse model of respiratory infection is the only normal adult model of reovirus infection causing disease and thus extends earlier studies of pathogenesis in the infant mouse to the adult. As reovirus disease is generally more severe in the suckling mouse, the adult mouse respiratory infection may more closely represent normal human infections. Although many normal cells are resistant to infection we describe virus type and host age dependency in cellular susceptibility to reovirus infection. Indeed the increased replication of reovirus strains in suckling mice and the age dependence of encephalitis are both consistent with the preferential replication in tumors that are poorly differentiated and rapidly growing cell types. Furthermore infection with T3D did not produce disease in immunocompetent adult animals which supports its safe use as a therapeutic virus in humans.

\section{Abbreviations}

T3D: Reovirus serotype 3 Dearing; T1L: Reovirus serotype 1 Lang; pfu: Plaque forming units; LD 50 : Median lethal dose; HRP: Horse radish peroxidase.

\section{Competing interests}

All of the authors declare that they have no competing interests with respect to the publication of this manuscript.

\section{Authors' contributions}

Conceived and planned experiments: EGB, SB, MS. Performed experiments: $L G, H L, M H, J M, E G B$. Supplied reagents: EGB, SB. Wrote and edited the manuscript: EGB, MS. All authors read and approve the final manuscript.

\section{Acknowledgements}

This work was funded by the Natural Sciences and Engineering Research Council of Canada to EGB.

\section{Author details \\ 'Department of Biochemistry, Microbiology and Immunology, Faculty of Medicine, University of Ottawa, 451 Smyth Rd, Ottawa, Ontario K1H 8M5, Canada. ${ }^{2}$ Emerging Pathogens Research Centre, University of Ottawa, 451 Smyth Rd, Ottawa, Ontario K1H 8M5, Canada. ${ }^{3}$ Department of Cellular and Molecular Medicine, Faculty of Medicine, University of Ottawa, 451 Smyth Rd, Ottawa, Ontario K1H 8M5, Canada. ${ }^{4}$ Program in Neuroscience, Ottawa Hospital Research Institute University of Ottawa, 451 Smyth Road, RGN \#1412, Ottawa, ON K1H 8M5, Canada. ${ }^{5}$ Division of Neurology, The Ottawa Hospital, University of Ottawa, 451 Smyth Road, RGN \#1412, Ottawa, ON K1H $8 \mathrm{M} 5$, Canada.}

Received: 27 July 2012 Accepted: 25 February 2013

Published: 1 March 2013

\section{Reference}

1. Jackson GG, Muldoon RL: Viruses causing common respiratory infection in man. IV. Reoviruses and Adenoviruses. J Infect Dis 1973, 128:811-866.

2. Rosen L, Hovis JF, Mastrota FM, Bell JA, Huebner RJ: Observations on a newly recognized virus (Abney) of the reovirus family. Am J Hyg 1960, 71:258-265.

3. Rosen $L$, Evans HE, Spickard A: Reovirus infections in human volunteers. Am J Hyg 1963, 77:29-37.
4. Tyler KL: Fields' virology. 4th edition. Philadelphia: Lippincott Williams \& Wilkins; 2001.

5. Norman KL, Lee PW: Reovirus as a novel oncolytic agent. J Clin Invest 2000, 105:1035-1038.

6. Coffey MC, Strong JE, Forsyth PA, Lee PW: Reovirus therapy of tumors with activated Ras pathway. Science 1998, 282:1332-1334.

7. Strong JE, Coffey MC, Tang D, Sabinin P, Lee PW: The molecular basis of viral oncolysis: usurpation of the Ras signaling pathway by reovirus. EMBO J 1998, 17:3351-3362.

8. Raine CS, Fields BN: Reovirus type 3 encephalitis-a virologic and ultrastructural study. J Neuropathol Exp Neurol 1973, 32:19-33.

9. Margolis G, Kilham L, Gonatas NK: Reovirus type 3 encephalitis: observations of virus-cell interactions in neural tissues. I. Light microscopy studies. Lab Invest 1971, 24:91-100.

10. Terheggen F, Benedikz E, Frissen PH, Brinkman K: Myocarditis associated with reovirus infection. Eur J Clin Microbiol Infect Dis 2003, 22:197-198.

11. Sherry B: Pathogenesis of reovirus myocarditis. Curr Top Microbiol Immunol 1998, 233:51-66.

12. Sherry B, Schoen FJ, Wenske E, Fields BN: Derivation and characterization of an efficiently myocarditic reovirus variant. J Virol 1989, 63:4840-4849.

13. Kauffman RS, Wolf $J$, Finberg R, Trier JS, Fields BN: The sigma 1 protein determines the extent of spread of reovirus from the gastrointestinal tract of mice. Virology 1983, 124:403-410.

14. Derrien M, Fields BN: Anti-interleukin-3 and anti-nerve growth factor increase neonatal mice survival to reovirus type 3 clone 9 per oral challenge. J Neuroimmunol 2000, 110:209-213.

15. Derrien $M$, Fields BN: Reovirus type 3 clone 9 increases interleukin-1alpha level in the brain of neonatal, but not adult, mice. Virology 1999, 257:35-44.

16. Bodkin DK, Fields BN: Growth and survival of reovirus in intestinal tissue: role of the L2 and S1 genes. J Virol 1989, 63:1188-1193.

17. Organ EL, Rubin DH: Pathogenesis of reovirus gastrointestinal and hepatobiliary disease. Curr Top Microbiol Immunol 1998, 233:67-83.

18. Majeski El, Paintlia MK, Lopez AD, Harley RA, London SD, London L: Respiratory reovirus $1 / \mathrm{L}$ induction of intraluminal fibrosis, a model of bronchiolitis obliterans organizing pneumonia, is dependent on T lymphocytes. Am J Pathol 2003, 163:1467-1479.

19. Majeski El, Harley RA, Bellum SC, London SD, London L: Differential role for $T$ cells in the development of fibrotic lesions associated with reovirus 1/L-induced bronchiolitis obliterans organizing pneumonia versus acute respiratory distress syndrome. Am J Respir Cell Mol Biol 2003, 28:208-217.

20. Bellum SC, Dove D, Harley RA, Greene WB, Judson MA, London L, et al: Respiratory reovirus $1 / L$ induction of intraluminal fibrosis. A model for the study of bronchiolitis obliterans organizing pneumonia. Am J Pathol 1997, 150:2243-2254.

21. London L, Majeski El, Paintlia MK, Harley RA, London SD: Respiratory reovirus $1 / L$ induction of diffuse alveolar damage: a model of acute respiratory distress syndrome. Exp Mol Pathol 2002, 72:24-36.

22. London L, Majeski El, Altman-Hamamdzic S, Enockson C, Paintlia MK, Harley $R A$, et al: Respiratory reovirus $1 / L$ induction of diffuse alveolar damage: pulmonary fibrosis is not modulated by corticosteroids in acute respiratory distress syndrome in mice. Clin Immunol 2002, 103:284-295.

23. Morin MJ, Warner A, Fields BN: Reovirus infection in rat lungs as a model to study the pathogenesis of viral pneumonia. J Virol 1996, 70:541-548.

24. Morin MJ, Warner A, Fields BN: A pathway for entry of retroviruses into the host through M cells of the respiratory tract. J Exp Med 1994, 180:1523-1527.

25. Nygaard RM, Golden JW, Schiff LA: Impact of host proteases on reovirus infection in the respiratory tract. J Virol 2012, 86:1238-1243.

26. Zou S, Brown EG: Stable expression of the reovirus mu2 protein in mouse $L$ cells complements the growth of a reovirus ts mutant with a defect in its M1 gene. Virology 1996, 217:42-48.

27. Schlossmacher MG, Shimura H: Parkinson's disease: assays for the ubiquitin ligase activity of neural Parkin. Methods Mol Biol 2005, 301:351-369.

28. Smeenk CA, Wright KE, Burns BF, Thaker AJ, Brown EG: Mutations in the hemagglutinin and matrix genes of a virulent influenza virus variant, A/FM/1/47-MA, control different stages in pathogenesis. Virus Res 1996, 44:79-95.

29. Haller BL, Barkon ML, Vogler GP, Virgin HW: Genetic mapping of reovirus virulence and organ tropism in severe combined immunodeficient mice: organ-specific virulence genes. J Virol 1995, 69:357-364. 
30. Sweet C, Smith H: Pathogenicity of influenza virus. Microbiol Rev 1980, 44:303-330

31. Smith $H$, Sweet $\mathrm{C}$ : Lessons for human influenza from pathogenicity studies with ferrets. Rev Infect Dis 1988, 10:56-75.

32. Kuiken $T$, van den Hoogen BG, van Riel DA, Laman JD, van Amerongen $G$, Sprong $L$, et al: Experimental human metapneumovirus infection of cynomolgus macaques (Macaca fascicularis) results in virus replication in ciliated epithelial cells and pneumocytes with associated lesions throughout the respiratory tract. Am J Pathol 2004, 164:1893-1900.

33. Machii K, Otsuka Y, Iwai H, Ueda K: Infection of rabbits with Sendai virus. Lab Anim Sci 1989, 39:334-337.

34. Wolf JL, Rubin DH, Finberg R, Kauffman RS, Sharpe AH, Trier JS, et al: Intestinal M cells: a pathway for entry of reovirus into the host. Science 1981, 212:471-472.

35. Helander A, Silvey KJ, Mantis NJ, Hutchings AB, Chandran K, Lucas WT, et al: The viral sigma1 protein and glycoconjugates containing alpha2-3 -linked sialic acid are involved in type 1 reovirus adherence to $M$ cell apical surfaces. J Virol 2003, 77:7964-7977.

36. Kilham L, Margolis G: Hydrocephalus in hamsters, ferrets, rats, and mice following inoculations with reovirus type I. I. Virologic studies. Lab Invest 1969, 21:183-188.

37. Tyler KL, McPhee DA, Fields BN: Distinct pathways of viral spread in the host determined by reovirus S1 gene segment. Science 1986, 233:770-774.

38. Tyler KL, Virgin HW, Bassel-Duby R, Fields BN: Antibody inhibits defined stages in the pathogenesis of reovirus serotype 3 infection of the central nervous system. J Exp Med 1989, 170:887-900

39. Matoba Y, Colucci WS, Fields BN, Smith TW: The reovirus M1 gene determines the relative capacity of growth of reovirus in cultured bovine aortic endothelial cells. J Clin Invest 1993, 92:2883-2888.

40. Margolis G, Kilham L: Hydrocephalus in hamsters, ferrets, rats, and mice following inoculations with reovirus type I. II. Pathologic studies. Lab Invest 1969, 21:189-198.

41. Tyler KL, Bronson RT, Byers KB, Fields B: Molecular basis of viral neurotropism: experimental reovirus infection. Neurology 1985, 35:88-92.

42. Hirasawa K, Nishikawa SG, Norman KL, Alain T, Kossakowska A, Lee PW: Oncolytic reovirus against ovarian and colon cancer. Cancer Res 2002, 62:1696-1701

43. Norman KL, Coffey MC, Hirasawa K, Demetrick DJ, Nishikawa SG, DiFrancesco LM, et al: Reovirus oncolysis of human breast cancer. Hum Gene Ther 2002, 13:641-652.

44. Wilcox ME, Yang W, Senger D, Rewcastle NB, Morris DG, Brasher PM, et al: Reovirus as an oncolytic agent against experimental human malignant gliomas. J Natl Cancer Inst 2001, 93:903-912.

doi:10.1186/1743-422X-10-67

Cite this article as: Gauvin et al: Respiratory infection of mice with mammalian reoviruses causes systemic infection with age and strain dependent pneumonia and encephalitis. Virology Journal 2013 10:67.

\section{Submit your next manuscript to BioMed Central and take full advantage of:}

- Convenient online submission

- Thorough peer review

- No space constraints or color figure charges

- Immediate publication on acceptance

- Inclusion in PubMed, CAS, Scopus and Google Scholar

- Research which is freely available for redistribution 